\title{
Secure Routing using Clustering Algorithm
}

\author{
Poonam V.Sadafal \\ SInhgad Institute of Technology \& science, Narhe, \\ Pune-41
}

\author{
R.H.Borhade \\ Assistant Professor \\ Smt. Kashibai Navale College of Engg., Vadgaon, \\ Pune-41
}

\begin{abstract}
ABSTRCAT
Wireless sensor network are build up of various autonomous sensors which are responsible for monitoring environmental or physical conditions, such as temperature, sound and pressure. And pass their data through the network to the sink node..There are various applications for wireless sensor network, and security is very important for many of them. Wireless sensor network has some key constraints such as limited energy resources, lack of infrastructure. All these constraints impose some security challenges. Wireless sensor network's development is originally motivated by military applications such as battlefield surveillance. Due to broadcast nature of transmission medium, also resource limitation on sensor nodes and their uncontrolled environments where they are left unattended, due to which these networks are susceptible to many attacks. Hence there is need of security mechanism in WSN. In this paper, secure DRINA algorithm is implemented for secure data transmission. Data routing in Network Aggregation (DRINA) is routing protocol which is designed for data aggregation in wireless sensor network. The main feature of DRINA is it provides best aggregation than the INFRA and SPT algorithms[1].
\end{abstract}

\section{Keywords}

Wireless Sensor Network (WSN), Data routing for in-network aggregation for WSNs (DRINA), Routing, Clustering, Security

\section{INTRODUCTION}

Security is important issue in wireless sensor network ,the development of wireless sensor network was originally motivated by military applications such as battlefield surveillance where the security is main issue. In military sensor network, attackers are attempting to gain and detect

as much as information about enemy movement. There are various techniques are used in order to achieve the security in wireless sensor network. Now days wireless sensor network widely used in many civilian application areas which include environment and healthcare applications, home automation, habitat monitoring and traffic control. Security plays a fundamental role in many wireless sensor network applications. In military applications, security is major issue which includes gaining and detecting as much as information about enemies movement. So in order to achieve security in wireless sensor network some security mechanism must be used. There various clustering algorithm is developed for wireless sensor network for effective data transmission. But in all this clustering algorithm security constrains is not considered. Data routing in Network Aggregation (DRINA) is routing protocol which is designed for data aggregation in wireless sensor network. The main feature of DRINA is it provides best aggregation than the INFRA and SPT algorithms. DRINA has some key aspects like no. of messages are less which required for setting up the routing tree, reliable data aggregation. The main goal of DRINA is to connect all nodes to sink node with shortest path while optimizing the data aggregation. However it does not have security mechanism for data transmission. As the information provided by the networks has been increased, there is a need for secure transmission of information. Several cryptographic and steganographic techniques are used in order to achieve security in wire sensor networks. In this project Secure DRINA algorithm is implemented. To achieve the security in existing DRINA algorithm, 'Public key cryptography' (RSA) algorithm is used

\section{DRINA: DATA ROUTING FOR IN- NETWORK AGGREGATION FOR WSNS.}

The main goal of DRINA is to build a routing tree and find out the shortest path which connects all source nodes to the sink, while maximizing the data aggregations. In DRINA following roles are consider for building the routing tree.

1. Collaborator: - It is node which detects an event and reports the collected data to the Coordinator node.

2. Coordinator: - It is collaborator node detects an event but after using election algorithm it become a coordinator. This node is responsible for aggregating the collected data received from other collaborator and send results to the sink node.

3. Relay node: This node which is in route between coordinator and sink node, and forwards the data to the sink

Sink Node: This node is interested in receiving the data from set of coordinator nodes \& collaborator node [1].

DRINA algorithm is divided into three phase.

- Building Hop tree from sensor nodes to the sink node.

- Forming Cluster and electing a cluster head among the collaborator which becomes a coordinator.

- Routing means forwarding data towards to a sink node.

\section{IMPLEMENTING SECURITY IN DRINA}

In all previous clustering algorithms until now security constraints is not considered. Hence there is challenge to perform security on clustering algorithm for security purpose. For example in military, security is main constraint whenever there is need of WSN in military .In this project security is implemented on existing clustering algorithm DRINA.

\section{SYSTEM MODEL}

Here first of all we are calculating the false data sent by sensing node to the sink node

Let $\mathrm{n}$ nodes are attacker in total $\mathrm{m}$ nodes so probability of node being attacker is given by

$\mathrm{P}(\mathrm{A})=\mathrm{n} / \mathrm{m}$ 
Assuming every attacker sends false data, if attacker is coordinator then all data go through attacker is false data, so false data sent is

Dfalse $=\mathrm{D}(\mathrm{FS})+\mathrm{D}(\mathrm{FC})$

Where

D(FS), Data false send by individual node

D (FC), data goes through attacking coordinator

False Data sent by individual node is given by

$\mathrm{D}(\mathrm{FS})=\mathrm{P}(\mathrm{A}) * \mathrm{SR}=(\mathrm{n} / \mathrm{m}) * \mathrm{SR}$

Where,

$\mathrm{SR}=$ sensing rate

Probability of attacker as coordinator is given by

$\mathrm{P}(\mathrm{FC})=\mathrm{P}(\mathrm{A}) * \mathrm{P}(\mathrm{C})$

Where $\mathrm{P}(\mathrm{C})$ is probability of being coordinator

$\mathrm{P}(\mathrm{C})=1 / \mathrm{N}$

Where $\mathrm{N}$ is number of neighbor

Putting (1) and (5) in (4)

$\mathrm{P}(\mathrm{FC})=\mathrm{n} / \mathrm{m} *(1 / \mathrm{N})=\mathrm{n} /(\mathrm{m} * \mathrm{~N})$

Probability of attacking coordinator on path of length 1 is given by

$\mathrm{Pt}(\mathrm{FC})=\mathrm{P}(\mathrm{FC}) * \mathrm{l}=\mathrm{nl} /(\mathrm{m} * \mathrm{~N})$

So false data send by coordinator (as data coming from

loyal node to attacking coordinator is also falsely forwarded)

$\mathrm{N}$

$\mathrm{D}(\mathrm{FC})=\mathrm{Pt}(\mathrm{FC}) * \sum((1-\mathrm{P}(\mathrm{A})) * \mathrm{SR})$

$\mathrm{K}=0$

$\mathrm{N}$

$\mathrm{D}(\mathrm{FC})=\mathrm{n} 1 /(\mathrm{m} * \mathrm{~N}) * \sum((1-\mathrm{n} / \mathrm{m}) * \mathrm{SR})$

$\mathrm{k}=0$

By putting (3) and (8) into (2) we will get total false data sent as

Dfalse $=$

$\mathrm{N}$

$$
(\mathrm{n} / \mathrm{m}) * \mathrm{SR}+(\mathrm{n} 1 / \mathrm{m} * \mathrm{~N}) * \sum((1-(\mathrm{n} / \mathrm{m})) * \mathrm{SR})
$$$$
\mathrm{k}=0
$$

This eq no. (9) Will give us amount of false data sent by coordinator.

In proposed method, data is encrypted before transmitting to sink node as

$\mathrm{DT}=\mathrm{E}(\mathrm{Kps}, \mathrm{E}(\mathrm{Kp}, \mathrm{SD}))$

Where,

$\mathrm{E}$ is public key encryption function

Kps is public key of sink node

$\mathrm{Kp}$ is private key of Sensing Node
Because of above encryption attacking coordinator node can't read data and can't send data with other nodes identity. Attacker nodes are still able to send false data, so total false data coming to sink is

Dfalse $=\mathrm{n} / \mathrm{m} * \mathrm{SR}$

This is improved than existing system.

\section{SIMULATION RESULT}

The simulation of the algorithm is performed using OMNET++.The nodes are placed randomly in given area. The simulation parameters are shown in Table 1.

Table 1

Simulation Parameter

\begin{tabular}{|l|l|}
\hline Parameters & Value \\
\hline Numbers of nodes & 20 \\
\hline Number of & 02 \\
\hline Number of sinks & 1 \\
\hline Initial Energy & $18720 \mathrm{~J}$ \\
\hline Transmission & $62 \mathrm{~mW}$ \\
\hline Receive power & $62 \mathrm{~mW}$ \\
\hline
\end{tabular}

Fig 5.2 shows the number of false packet detected by nodes. The no. of false packet detected by only those node which are in the already established routing route or which are closer to attacking node.

\begin{tabular}{|c|c|c|}
\hline \multicolumn{3}{|c|}{$\begin{array}{l}\text { Browse Data } \\
\text { Here you can see all data that come from the files specified in the inputs page. }\end{array}$} \\
\hline \multicolumn{3}{|c|}{ All (19/19) Vectors $(19 / 19)$ Scalars (0/0) Histograms (0/0) } \\
\hline 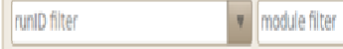 & 1) statiste name filter & 77 \\
\hline Name & Value & \\
\hline \multicolumn{3}{|l|}{ - DGenteral: } \\
\hline \multicolumn{3}{|l|}{ 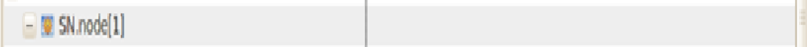 } \\
\hline 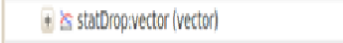 & $0.0(1000)$ & \\
\hline \multicolumn{3}{|l|}{ - 䁫 SN nodel[2] } \\
\hline + 管 statDropiector (vector) & $0.0(1000)$ & \\
\hline \multicolumn{3}{|l|}{ - 资 $5 \mathrm{~N} . n \operatorname{node}[3]$} \\
\hline + Es statbirop:vector (vector) & $0.0(1002)$ & \\
\hline \multicolumn{3}{|l|}{$=$ - 5 SN.node(4) } \\
\hline + s statbropestor (vestor) & 245.89043824701196 (1004) & \\
\hline
\end{tabular}

Fig 5.2. False Packet detected by nodes

Fig $5.3 \& 5.4$ shows the comparative result of existing DRINA and secure DRINA in terms of energy consumed by each node. This result shows that energy consumed by nodes in existing DRINA algorithm is not much varying in secure DRINA. 


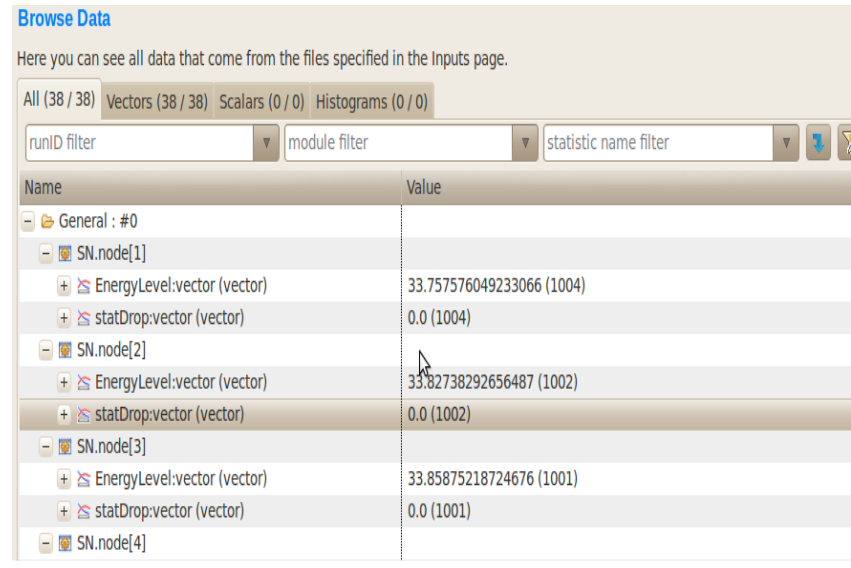

Fig 5.3. Energy level of nodes in Existing DRINA

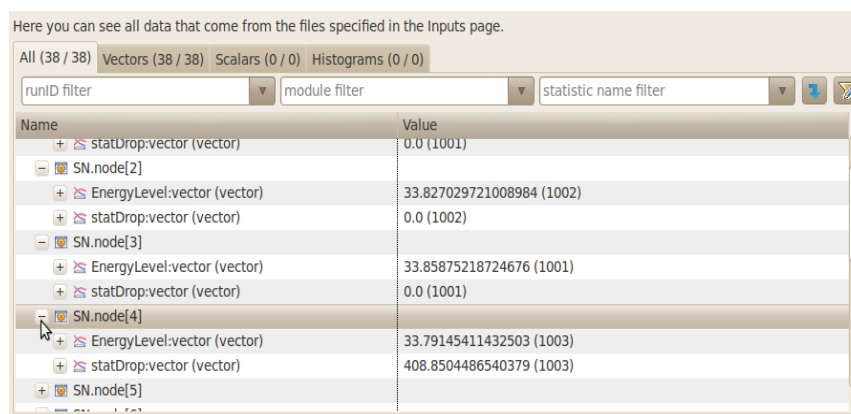

Fig 5.4.Energy level of nodes in Secure DRINA.

\section{CONCLUSION}

Security is main constraint in wireless sensor network. So using RSA and SHA algorithm it is possible to provide security to any routing algorithm. In this paper security is provided to DRINA clustering algorithm in order to achieve two main goals of security which is nothing but confidentiality and Authentication.

\section{REFERENCES}

[1] DRINA: A Lightweight and Reliable Routing Approach for in-Network Aggregation in Wireless Sensor Networks Leandro Villas1 2, Azzedine Boukerche1, Heitor S. Ramos1 , 2, Horacio A. B. F. de Oliveira3, Regina B. de Araujo4 and Antonio A. F. Loureiro2 2012 IEEE.
[2] L. Villas, A. Boukerche, R. B. de Araujo, and A. A. F. Loureiro, "Highly dynamic routing protocol for data aggregation in sensor networks," in Proceedings of the The IEEE symposium on Computers and Communications, ser. ISCC '10. Washington, DC, USA: IEEE Computer Society, 2010,

[3] H. S. AbdelSalam and S. Olariu, "A lightweight skeleton construction algorithm for self- organizing sensor networks." in ICC. IEEE, 2009, pp. 1-5. [Online]. Available: http://dblp.uni-trier.de/db/conf/icc/icc2009

[4] G. Anastasi, M. Conti, M. Francesco, and A. Passarella "Energy conservation in wireless sensor networks: A survey," Ad Hoc Networks, vol. 7, no. 3, pp. 537-568, May 2009.

[5] Boukerche, Algorithms and Protocols for Wireless Sensor Networks. Wiley-IEEE Press, 2008.

[6] Ameer Ahmed Abbasi Mohamed Younis "A survey on clustering algorithms for wireless sensor networks", Department of Computing, Al-Hussan Institute of Management and Computer Science,Dammam 31411, Saudi Arabia Department of Computer Science and Electrical Engineering, University of Maryland, Baltimore County, Baltimore, MD 21250, USA ,21 June 2007.

[7] Boukerche, R. B. Araujo, and L. Villas, "Optimal route selection for highly dynamic wireless sensor and actor networks environment," in MSWiM , Proceedings of the 10th ACM Symposium on Modeling, analysis, and simulation of wireless and mobile systems. NewYork, NY, USA: ACM, 2007.

[8] E. F. Nakamura, H. A. B. F. de Oliveira, L. F. Pontello, and A. A. F. Loureiro, "On demand role assignment for event-detection in sensor networks," in ISCC '06: Proceedings of the 11th IEEE Symposium on Computers and Communications. Washington, DC, USA: IEEE Computer Society, 2006.

[9] Dirk WESTHOFF, Joao GIRAO, "Security Solutions for Wireless Sensor Networks" Amardeo SARMA NEC TECHNICAL JOURNAL Vol.1 No.3/2006.

[10] S. Olariu, Q. Xu, and A. Zomaya, "An energy-efficient self-organization protocol for wireless sensor networks," in Intelligent Sensors, Sensor Networks and Information Processing Conference (ISSNIP). Melbourne,Australia: IEEE, December 2004. 